\title{
BOAO PHOTOMETRIC SURVEY OF GALACTIC OPEN CLUSTERS. II. Physical Parameters of 12 Open Clusters
}

\author{
H. B. Ann and S. H. Lee \\ Department of Earth Science, Pusan National University, Pusan 609-735, Korea \\ (hbann@hyowon.pusan.ac.kr, shlee@jupiter.es.pusan.ac.kr) \\ H. Sung and M. G. Lee \\ Astronomy Program, SEES, Seoul National University, Seoul 151-742, Korea \\ (sungh@astro.snu.ac.kr, mglee@astrog.snu.ac.kr) \\ S. -L. Kim, M. Y. Chun, Y. -B. Jeon, B. -G. Park, and I. -S. Yuk \\ Korea Astronomy Observatory, Taejeon, 305-348, Korea (slkim@kao.re.kr, \\ mychun@kao.re.kr, ybjeon@boao.re.kr, bgpark@boao.re.kr, yukis@kao.re.kr)
}

Received — 


\begin{abstract}
We have initiated a long-term project, the BOAO photometric survey of open clusters, to enlarge our understanding of galactic structure using $U B V I \mathrm{CCD}$ photometry of open clusters which have been little studied before. This is the second paper of the project in which we present the photometry of 12 open clusters. We have determined the cluster parameters by fitting the Padova isochrones to the color-magnitude diagrams of the clusters. All the clusters except for Be 0 and NGC 1348 are found to be intermediate-age to old (0.2 - 4.0 Gyrs) open clusters with a mean metallicity of $[\mathrm{Fe} / \mathrm{H}] \approx 0.0$.
\end{abstract}

Subject headings: Open clusters and associations : general - Open clusters and associations : individual (Be 104, Be 60, King 15, NGC 381, Be 64, King6, NGC 1348, Be 12, Be 70, Be 23, NGC 2259 and NGC 2304) - photometry : distance, age

\title{
1. INTRODUCTION
}

Open clusters are an ideal tool for tackling many astrophysical problems such as star formation processes, stellar evolution and dynamical evolution of stellar systems. To date there are about 1200 known galactic open clusters in the Catalog of Open Cluster Data (Lyngå 1987; hereafter COCD). Owing to the high quantum efficiency, good linearity and large dynamic range of modern CCDs, accurate color-magnitude diagrams (CMD) of a large number of open clusters have been obtained to derive their basic physical parameters (distance, interstellar reddening, age and metallicity). Current understanding of the galactic disk has greatly benefited from several recent systematic CCD observations of young and old open clusters (Phelps \& Janes 1993; Janes \& Phelps 1994). 
However, more than half of the known galactic open clusters are as yet virtually unstudied. All that we know about the majority of the open clusters are their positions and angular diameters, which are often inaccurate in many cases. To date only one third of the known open clusters have been studied in detail to derive their basic parameters. Moreover, more than half of the well studied clusters belong to Trumpler richness class $m$ and $r$, while most of the unstudied clusters are classified as $p$. Thus, present knowledge of open clusters is considered to be heavily biased by the properties of rich open clusters.

To understand the nature of open clusters and the structure of the galactic disk as a whole, we have started a comprehensive photometric survey of open clusters which have been little studied before, using the $1.8 \mathrm{~m}$ telescope at Bohyunsan Optical Astronomy Observatory (BOAO) in Korea. The primary goal of the present survey is to obtain accurate CMDs of the unstudied clusters using UBVI CCD photometry and to derive cluster parameters such as distance and age. We selected 343 clusters by the declination criterion of $\delta>-20^{\circ}$. They comprise about half of the clusters with unknown distance and age in COCD. A more detailed description of the present survey was given by Ann et al. (1999).

This is the second paper of our survey project. Here, we present the results of the photometric survey of 12 open clusters observed during the observing runs in 1998 and 1999. In $\S 2$, we describe the observations and data reduction. The physical parameters of the observed clusters are presented in $\S 3$ and a brief summary and discussion are given in the last section. 


\section{OBSERVATIONS}

We observed 12 candidate clusters of the BOAO photometric survey in December 1998. The basic parameters of the observed clusters (Lyngå 1987) are given in Table 1. The photometric observations were made using the SITe $2048 \times 2048$ CCD camera with Johnson-Cousins $U B V I$ filters attached to the F/8 cassegrain focus of the BOAO $1.8 \mathrm{~m}$ telescope. The pixel size of the CCD is $24 \mu \mathrm{m}$ corresponding to $0 . .^{\prime \prime} 34$ on the sky. The gain and readout noise of the CCD are 1.8 electrons/ADU and 7 electrons, respectively. We have obtained images of the central $11 .^{\prime} 6 \times 11 .^{\prime} 6$ regions of each cluster with a pair of long and short exposures for each filter. We observed several $U B V I$ standard stars in Landolt (1992)'s catalog for the calibration of our photometry during the observing run. However, owing to non-photometric conditions and difficulty in transforming the $U-B$ colors to the standard system, we re-observed the clusters in a short exposure with the SAAO version of Landolt standard stars in the equator (Menzies et al. 1991) and blue and red standard stars (Kilkenny et al. 1998) on a photometric night during the observing run in October

1999. The observed standard stars (or regions) are SA 112, SA 114, SA 93, SA 99, BD -11 162, GL 876 and GL 273. Table 2 lists the observational log.

Grayscale maps of the $V$-band images of the clusters taken with long exposures are displayed in Fig. 1. In most cases, the cluster field is a small part of the observed frame. Solid circles in Fig. 1 represent the angular diameters of the clusters given in the COCD whose centers were determined by visual inspection of the images. We obtained photometric diagrams of the clusters for the stars located within the circles.

We have followed standard CCD reduction techniques using IRAF/CCDRED for the preprocessing of the images obtained. This includes the subtraction of bias levels with overscan correction, trimming and flat-fielding. We used IRAF/DAOPHOT to obtain instrumental magnitudes of the stars. We applied aperture corrections to the instrumental 
magnitudes of cluster stars obtained from Point Spread Function (PSF) fitting, using the instrumental magnitudes obtained with an aperture radius of $7^{\prime \prime}$.

The transformation of the instrumental system to the standard system was made using the following equations (see the similar transformation for the $40^{\prime \prime}$ telescope at Siding Spring Observatory: (Sung \& Bessell 2000)):

$$
V=v-k_{V} X-0.046(B-V)+\zeta_{V}
$$

or

$$
\begin{gathered}
V=v-k_{V} X-0.046(V-I)+\zeta_{V}^{\prime} \quad \text { for } V-I<1.5 \\
V=v-k_{V} X+\zeta_{V}^{\prime \prime} \quad \text { for } V-I \geq 1.5 \\
I=i-k_{I} X-0.017(V-I)+\zeta_{I} \quad \text { for } V-I<0.6 \\
I=i-k_{I} X+0.083(V-I)+\zeta_{I}^{\prime} \quad \text { for } V-I \geq 0.6 \\
B=b-k_{B} X+0.112(B-V)+\zeta_{B} \\
U=u-k_{U} X+f(B-V)+\zeta_{U}
\end{gathered}
$$

where uppercase characters, lowercase characters, $k, \mathrm{X}$ and $\zeta$ represent standard magnitudes, instrumental magnitudes, extinction coefficients, airmass, and zero points, respectively. In the transformation of the $U$ magnitude, a non-linear function, $f(B-V)$, is involved. The function $f(B-V)$ allows the correction of redder $(U-B)$ colors, probably due to a red-leak in the $U$ filter (Bessell 2001). It increases linearly up to $(B-V) \approx 0.8$, but shows a non-linear increase for redder stars. This correction dominated the other factors, and we did not take into account the $(U-B)$ term in the $U$ transformation. The extinction coefficients derived from standard star photometry are $k_{U}=0.485 \pm 0.024, k_{B}=0.232 \pm$ $0.035, k_{V}=0.142 \pm 0.023$ and $k_{I}=0.058 \pm 0.027$. The final transformation errors were between $0.021 \mathrm{mag}$ in $V$ and $0.038 \mathrm{mag}$ in $U$. The instrumental magnitudes obtained from the observations in non-photometric conditions during the December run were transformed 
to the standard system using the observations of the October run. The photometric data will be presented in the electronic AJ.

We calculate the external observational errors of the photometry of the cluster stars using the pair of exposures in the 1998 December run and the short exposures in the 1999 October run. Fig. 2 shows the resulting observational errors as a function of $V$ magnitude. The error bars in Fig. 2 are the standard deviations of the observational errors for the stars in each magnitude bin. As shown in Fig. 2, the observational errors for stars fainter than $V \approx 14$ increase with increasing magnitude, while the observational errors for stars brighter than $V \approx 14$ are nearly constant with relatively large uncertainties due to the small number of stars in the magnitude bins.

\section{RESULTS}

We have determined the basic physical parameters of the 12 open clusters by analyzing the color-color diagrams and CMDs of the clusters. Photometric diagrams of the clusters are derived from the photometry of stars inside the angular diameters (Lyngå 1987) plotted in Fig. 1. To achieve improved accuracy in the derived parameters, we used stars with DAOPHOT errors less than 0.1 mag. In addition, due to large errors in $U$, the $(U-B)$ vs $(B-V)$ diagrams were constructed using stars brighter than $V \approx 17$. In general, as shown in Fig. 2, the total observational errors are quite large $\left(\sigma_{v} \approx 0.03\right)$ even for the brightest stars. However, the present photometry is good enough to derive the cluster parameters using isochrone fittings.

We have estimated the reddening of the clusters from $(U-B)$ vs $(B-V)$ diagrams, by comparing theoretical ZAMS of the Padova isochrones (Bertelli et al. 1994) with the observed distributions of cluster stars. To do this, we assumed a slope of 
$E(B-V) / E(U-B)=0.72$ (Johnson \& Morgan 1953), a total to selective extinction ratio $R_{V}=3.0$ and $E(V-I)=1.25 E(B-V)$ (Dean, Warren \& Cousins 1978). The advantage of using theoretical ZAMS is the ability to take into account the metallicity effect on the stellar distribution in the color-color diagrams. Because the metallicity of clusters is not known beforehand, we assumed the solar abundance as the cluster abundance unless the stellar distributions in the color-color diagrams and CMDs can not be described by the solar abundance isochrones.

We used the Padova isochrones (Bertelli et al. 1994) in the isochrone fittings. We put the greatest weight on the stars in the turnoff regions and giant branches, if present, since the lower main sequences are more heavily contaminated by field stars. We attempted to fit the lower envelope of the main sequence, considering the photometric errors. The best fit solutions were obtained by visual inspections of the CMDs with the trial fittings. We present the results of the best fits in Fig. 3 and the resulting cluster parameters are given in Table 3.

Since we put the greatest weight on the stars in the regions which are supposed to be the giant branches of some clusters, the memberships of these stars are crucial to the derived cluster parameters. To see whether these stars are cluster giants or not, we plotted cleaned CMDs in Fig. 4. To obtain the cleaned CMDs, we statistically removed field stars from the cluster CMDs by finding stars nearest to the field stars with a photometric box of \pm 0.25 in $V$ and \pm 0.1 in $(B-V)$. Because we did not observe field region of each cluster, we constructed the CMDs of field stars by selecting stars randomly from the regions outside the radii that are two times larger than the cluster radii in the same CCD images, except for Be 70 for which field stars are selected from the region outside 1.5 times larger than the cluster radius due to the larger angular size of the cluster. As shown in Fig. 4, giant stars are left over in the cleaned CMDs. This result strongly suggests that many stars in the red 
giants locus could be the members of the cluster. In the following, we briefly describe the properties of the individual clusters.

\subsection{Berkeley 104}

Be 104 is located to the south of a very bright star, HD225094. As indicated by its Trumpler class of $I I 1 p$, Be 104 is one of the most poorly defined clusters. Due to the paucity of members and the heavy contamination by field stars, the CMDs of Be 104 show a very broad main sequence which is not much different from that of the field star CMDs near the cluster. Thanks to the presence of a few giants which are thought to be cluster members as they appear in the cleaned CMD in Fig.4, we could derive the cluster parameters by fitting the isochrones to the stellar distributions in the CMDs. The resulting cluster parameters are $(m-M)_{0}=13.2 \pm 0.2, E(B-V)=0.45 \pm 0.05, \log (\mathrm{t}[\mathrm{yrs}])=8.9 \pm 0.1$, and $[\mathrm{Fe} / \mathrm{H}]$ $=0.07$ dex. Most of the stars brighter than the turnoff point of the main-sequence stars are considered to be field stars. However, there seem also to be a few blue straggler candidates according to their positions on the CMDs.

\section{2. $\quad$ Berkeley 60}

Be 60 is also a poorly defined cluster with a Trumpler class of III1p. As shown in Fig. 1, there are several bright stars in the field of Be 60 most of which seem to be foreground stars. However, the CMDs of the stars in the central region of Be 60 show a well defined main sequence which permits a reasonably good isochrone fitting. The resulting best fit isochrone gives the cluster parameters as $(m-M)_{0}=13.2 \pm 0.2, E(B-V)=0.86 \pm 0.05$, $\log (\mathrm{t}[\mathrm{yrs}])=8.2 \pm 0.2$ and $[\mathrm{Fe} / \mathrm{H}]=0.07$ dex. The two brightest red stars near $(B-V)=1.6$ are not plotted in the $V$ vs $(V-I)$ CMD due to saturation in the $I$ image. 


\section{3. $\quad$ King 15}

As shown in Fig. 1, King 15 shows the typical properties of $I V 2 p$ clusters, which are characterized by a small number of member stars and no central concentration. However, as can be seen in Fig. 3, the stellar distributions in the CMDs of the cluster are well represented by the isochrone of $\log (\mathrm{t}[\mathrm{yrs}])=8.4 \pm 0.1$ and $[\mathrm{Fe} / \mathrm{H}]=0.07$ dex with a distance modulus and reddening of $(m-M)_{0}=12.5 \pm 0.4$ and $E(B-V)=0.70 \pm 0.12$, respectively. Phelps \& Janes (1994) derived a slightly smaller distance modulus, $(m-M)_{0}=12.29$ and smaller reddening, $E(B-V)=0.46$ for King 15 . Since they had no $U$-band photometry, their reddening estimate was obtained by fitting the Schmidt-Kaler (1982)'s ZAMS to the stellar distribution in the $V$ vs $(B-V)$ CMD. They estimated the age of King 15 to be $\sim 3$ Gyr from the color of the earliest spectral type star on the main-sequence $\left((B-V)_{o}=0.19\right)$. The reason for the large discrepancy between the age derived from the isochrone fittings and that found by Phelps \& Janes (1994) is due to the fact that they considered stars brighter than $V \approx 15$ to be field stars, while we consider them as cluster members according to their positions in the CMDs as well as spatial position in the cluster field.

As shown in Fig. 4, the presence of stars brighter than $V \approx 15$ in the cleaned CMD support the assumption that $V \sim 14$ are cluster members. However, it is quite natural for them to believe the stars brighter than $V \approx 15$ to be field stars since their CMD is heavily populated by field stars due to the larger field compared to ours. They constructed the CMD from the stars in a field of $11 .^{\prime} 6 \times 11 .^{\prime} 6$, which is nearly the same field as that of the total CCD image for King 15 in Fig. 1 . If stars brighter than $V \approx 15$ are member stars of King 15, there is a large gap in the upper main sequence of the cluster. 


\subsection{NGC 381}

NGC 381 is an intermediate-age open cluster with a Trumpler class of $I I I 1 \mathrm{~m}$. There seem to be no giant stars in the cluster probably due to its small number member stars. The main sequence of the cluster is well defined in the CMDs although there are a large number of field stars in the direction of NGC 381. Owing to the foreground and background field stars, the color-color diagram of NGC 381 is quite complicated. However, it is not difficult to determine the cluster reddening by fitting the theoretical ZAMS to the distribution of the main-sequence stars in the color-color diagram because they are well isolated from the field stars. As shown Fig.3, the isochrone of $\log (\mathrm{t}[\mathrm{yrs}])=8.5 \pm 0.1$ and $[\mathrm{Fe} / \mathrm{H}]=0.07$ dex fits well the stellar distribution in the CMDs of NGC 381 with a distance modulus and reddening of $(m-M)_{0}=10.3 \pm 0.3$ and $E(B-V)=0.40 \pm 0.10$, respectively. The present estimates of the distance modulus and reddening of the cluster are in good agreement with those determined by Phelps \& Janes (1994) but their age estimate of $\log (\mathrm{t}[\mathrm{yrs}])=9.04$,

obtained using the isochrone of Maeder \& Meynet (1991), is about a factor of three larger than ours.

\subsection{Berkeley 64}

Be 64 shows the typical morphology of a Trumpler class $I I 1 \mathrm{~m}$ cluster which is characterized by a slight concentration of member stars of similar brightness with medium richness. Because the color-color diagram does not show any clear cluster sequences due to the small number of bright stars with small photometric errors in $U$, we adopt $E(B-V)=1.05$ (Pandey et al. 1997) as the cluster reddening. The CMDs of Be 64 show a quite broad main sequence due to contamination by field stars However, the presence of giant stars, most of which are thought to be cluster members from the cleaned CMD in Fig. 4, makes it possible to fit the isochrones to the stellar distributions in the CMDs 
unambiguously. As shown in Fig. 3, the adopted isochrone of $\log (\mathrm{t}[\mathrm{yrs}])=9.0 \pm 0.1$ and $[\mathrm{Fe} / \mathrm{H}]=-0.61$ dex with $(m-M)_{0}=13.0 \pm 0.3$ and $E(B-V)=1.05 \pm 0.15$ fits the stellar distribution in the CMDs quite well. Our estimates of the distance modulus and age are in good agreement with those of Pandey et al. (1997).

\section{6. $\quad$ King 6}

King 6 is a nearby cluster with a Trumpler class of $I I 2 m$. As shown in Fig. 1, the angular diameter of the cluster is comparable to the size of the observed field because of its proximity. The CMDs of King 6 show a well defined main sequence with a relatively small number of field stars, especially in the upper main sequence. However, the lower main sequence, fainter than $V \sim 18$, seems to be contaminated by field stars. Although there seems to be a clear sequence in Fig. 3, the stellar distributions in the color-color diagram of King 6 can not be fitted by the theoretical ZAMS with a single reddening value. The stars fainter than $V \sim 16$ seem to have a smaller reddening of $E(B-V) \approx 0.4$, while the stars brighter than $V \sim 13$ appear to have a larger reddening of $E(B-V) \approx 0.6$. Thus, we adopted a mean value of $E(B-V)=0.5 \pm 0.10$ as the reddening of King 6. This is the reason why the stellar distributions in the color-color diagram show a relatively poor match with the theoretical ZAMS which is shifted by a reddening value of $E(B-V)=0.5$. However, as shown in Fig. 3, the isochrone of $\log (\mathrm{t}[\mathrm{yrs}])=8.4 \pm 0.1$ and $[\mathrm{Fe} / \mathrm{H}]=0.46$ dex, shifted by a distance modulus and reddening of $(m-M)_{0}=9.7 \pm 0.4$ and $E(B-V)=0.5 \pm 0.10$, respectively, gives a pretty good match with the stellar distributions in the CMDs of the cluster. There is a slight indication of the presence of the binary sequence above the ZAMS. 


\subsection{NGC 1348}

NGC 1348 is the youngest among the observed clusters and is relatively well recognized due to the absence of field stars comparable in brightness to the bright members of the cluster, although the cluster stars do not show any obvious central concentration. This is the reason why NGC 1348 is classified as Trumpler class III2m. As shown in Fig. 3, the color-color diagram of NGC 1348 shows a well defined sequence which is well matched with the theoretical ZAMS of the solar abundance shifted by $E(B-V)=1.02$. The isochrone fittings to the stellar distributions in the CMDs give the cluster parameters of $\log (\mathrm{t}[\mathrm{yrs}])$ $=8.1 \pm 0.1,(m-M)_{0}=11.3 \pm 0.3, E(B-V)=1.02 \pm 0.05$ and $[\mathrm{Fe} / \mathrm{H}]=0.07$ dex, respectively. The brightest star near $B-V=2.4$ is saturated in the $I$ image and is omitted in the $V$ vs $(V-I)$ diagram.

\subsection{Berkeley 12}

Be 12 is an old cluster with a Trumpler class of $I I 1 \mathrm{~m}$. Although Be 12 is located in the direction of a rich star field, it can be well distinguished from field stars due to the concentration of the member stars. The CMDs of the cluster show a broad main sequence caused by heavy contamination by field stars. The color-color diagram of Be 12 also shows no clear sequence due to the lack of a sufficient number of cluster main-sequence stars that are bright enough to have small photometric errors in $U$ as well as the contamination by

field stars. The presence of blue straggler stars which are located in the bright extension of the main sequence of Be 12 may contribute to the large scatter in the color-color diagram. However, as shown in Fig. 3, the isochrone of $\log (\mathrm{t}[\mathrm{yrs}])=9.6 \pm 0.1$ and $[\mathrm{Fe} / \mathrm{H}]=0.07$ dex fits the main sequence together with the giant branch quite well with a distance modulus and reddening of $(m-M)_{0}=12.5 \pm 0.5$ and $E(B-V)=0.7 \pm 0.15$, respectively. The old age of the cluster supports the existence of blue straggler stars based on the presence of 
stars brighter and bluer than the turnoff point of the cluster main sequence because such stars are more frequently found in old open clusters (Ahumada \& Lapasset 1995)

\section{9. $\quad$ Berkeley 70}

Be 70 is located in a direction toward which a large number of foreground stars are located. The CMDs of the cluster show a broad main sequence due to heavy contamination by field stars. The color-color diagram appears to be complicated due to the field star

contamination, but we could determine the reddening of the cluster by fitting the theoretical ZAMS to the distributions of upper main-sequence stars and populous giant stars which constitute a well defined cluster sequence in the CNDs. The broad sequence around $(B-V) \approx 0.7$ in the color-color diagram is due to the field star population, whose mean reddening is much smaller than that of the cluster. The heavy contamination of field stars is apparent from the cleaned CMD of Be 70 in Fig. 4. However, some of the bright blue stars above the turnoff point of the main sequence of Be 70 may be blue straggler stars from their presence in the cleaned CMD of the cluster. Owing to the well developed giant branch and the highly populated main sequences, it is not difficult to fit the isochrones to the stellar distributions in the CMDs. The resulting cluster parameters are $\log (\mathrm{t}[\mathrm{yrs}])$ $=9.6 \pm 0.1,[\mathrm{Fe} / \mathrm{H}]=-0.32 \mathrm{dex},(m-M)_{0}=13.1 \pm 0.3$ and $E(B-V)=0.48 \pm 0.10$.

\subsection{Berkeley 23}

Be 23 is located near the direction of the anti-galactic center direction. As indicated by its Trumpler class of $I I I 1 \mathrm{~m}$, it shows no strong central concentration but can be identified by its relatively dense population compared to that of the field stars. Most of the brightest stars above the turnoff point are considered to be foreground stars because 
the CMD of a nearby field region shows similar stellar distributions. However, there is also a possibility that some of the stars that are brighter and bluer than the turnoff of the main sequence are blue straggler stars. We derived the cluster parameters by fitting the isochrone of $\log (\mathrm{t}[\mathrm{yrs}])=8.9 \pm 0.1$ and $[\mathrm{Fe} / \mathrm{H}]=0.07$ to the stellar distribution, especially near the turnoff, with a distance modulus and reddening of $(m-M)_{0}=14.2 \pm 0.3$ and $E(B-V)=0.40 \pm 0.05$, respectively. The theoretical ZAMS of the solar abundance shifted by the adopted reddening of $E(B-V)=0.40$ describes well the distribution of upper main-sequence stars in the color-color diagram. Most of the forground stars seems to be affected by negligible reddening.

\subsection{NGC 2259}

NGC 2259 is a well detached system of Trumpler class II1p. Most of the bright stars in the direction of NGC 2259 are cluster main-sequence stars near the main-sequence turnoff. There seems to be little contamination by field stars in the upper main sequence but the lower main sequence is somewhat contaminated by field stars. Owing to the proximity of the cluster, the main-sequence stars bluer than $(B-V) \approx 1.0$ are bright enough to produce a clear sequence in the color-color diagram. Thus, we derived the cluster reddening by fitting the theoretical ZAMS to the distribution of bright main-sequence stars in the color-color diagram. We applied the isochrone fittings to the stellar distributions in the CMDs of NGC 2259 with the reddening derived from the ZAMS fitting. As shown in Fig. 3 , the isochrone of $\log (\mathrm{t}[\mathrm{yrs}])=8.5 \pm 0.1$ and $[\mathrm{Fe} / \mathrm{H}]=0.07$ dex with a distance modulus and reddening of $(m-M)_{0}=12.6 \pm 0.3$ and $E(B-V)=0.59 \pm 0.05$, respectively, matches well with the stellar distributions in the CMDs of NGC 2259. 


\subsection{NGC 2304}

NGC 2304 is located in the direction of the anti-galactic center, slightly above the galactic plane. It is well distinguished from the field stars. The color-color diagram shows a well defined cluster sequence which is nicely matched with the theoretical ZAMS of $[\mathrm{Fe} / \mathrm{H}]$ $=-0.32$ shifted by $E(B-V)=0.10$. However, as shown in Fig. 3, the main sequence, especially the lower main sequence, is somewhat contaminated by field stars. The stellar distributions in the CMDs of NGC 2304 show the typical characteristics of intermediate-age open clusters. The stars near the giant branch seem to be cluster members since all these stars are present in the cleaned CMD of NGC 2304 in Fig. 4. The isochrone of $\log (\mathrm{t}[\mathrm{yrs}])$ $=8.9 \pm 0.1$ and $[\mathrm{Fe} / \mathrm{H}]=-0.32$ dex with $(m-M)_{0}=13.0 \pm 0.1$ and $E(B-V)=0.10 \pm 0.02$ fits well the stellar distribution in the CMDs of NGC 2304. There seems to be a binary sequence above the ZAMS.

\section{DISCUSSION AND SUMMARY}

We have determined the distances, reddenings, ages and metallicities of 12 target clusters by isochrone fittings complemented by ZAMS fittings for the reddening estimates. To our best knowledge, no known cluster parameters were previously derived for any of the clusters except for King 15, NGC 381, and Be 64. Comparison of the cluster parameters derived from the present photometry with those found by other researchers for King 15, NGC 381 and Be 64 (Phelps \& Janes 1994; Pandey et al. 1997) shows that our estimates are in good agreement with independent studies.

The mean interstellar absorption derived from the reddening of the 12 open clusters is $0.72 \pm 0.51 \mathrm{mag} \cdot \mathrm{kpc}^{-1}$, which is slightly smaller than the mean interstellar absorption of

the galactic disk, $1.0 \mathrm{mag} \cdot \mathrm{kpc}^{-1}$, derived from COCD (Lyngå 1987). The smaller mean 
interstellar absorption reflects the general characteristics of the interstellar reddening toward the anti-galactic center, and the large standard deviation is due to the inhomogeneous distribution of the interstellar clouds (Lucke 1978). Be 23 and NGC 2304, which are located in a direction very close to the anti-galactic center, show extremely small interstellar absorptions of $0.17 \mathrm{mag} \cdot \mathrm{kpc}^{-1}$ and $0.08 \mathrm{mag} \cdot \mathrm{kpc}^{-1}$, respectively, while King 6 and NGC 1348, which are located in the direction of the Cassiopeia-Perseus region (Deutschman, Davis, \& Schild 1976), show large interstellar absorptions of $1.72 \mathrm{mag} \cdot \mathrm{kpc}^{-1}$ and 1.68 mag $\cdot \mathrm{kpc}^{-1}$, respectively. The large interstellar absorption in this direction might be due to the dense interstellar cloud close to the Sun (Deutschman, Davis, \& Schild 1976).

The apparent gap in the main sequence of King 15 seems to be a real feature because the stars brighter than $V \approx 15$ are thought to be cluster members. Thus, the age of King 15 is $\sim 250$ Myr, which is much smaller than that suggested by Phelps \& Janes (1994). However, the gap near $V \sim 14$ is not a physical gap related to stellar evolution but is caused by random statistical fluctuations due to the small number of member stars. King 15 is too young for the cluster to have a main-sequence gap similar to those of NGC 2420 (Anthony-Twarog et al. 1990; Lee, Ann, \& Kang 1999) and NGC 2243 (Bonifazi et al. 1990), which are thought to be related to the rapid hydrogen exhausting phase of the convective core (Demarque, Sarajedini, \& Guo 1994). The gap in King 15 is similar to those of Be 7, NGC 637, and Be 62 (Phelps \& Janes 1993), all of which are thought to be caused by statistical fluctuations in the high mass part of the IMFs (Scalo 1986).

We have found blue straggler candidates in the four open clusters, Be 104, Be 12, Be 70, and Be 23, which have ages greater than 800 Myr. There is a tendency for older clusters to have more blue straggler stars, similar to that found by Ahumada \& Lapasset (1995).

Our results for the 12 clusters presented in this study along with the results of Ann et al. (1999) are expected to be very useful for the investigation of the open cluster system in 
our Galaxy. Our survey project is still in progress and we plan to present a study of more open clusters in the near future.

The authors would like to thank to KAO for the allocation of telescope times. The work by H.B.Ann was supported in part by PNU grant 1999-2003. 


\section{REFERENCES}

Ahumada, J., \& Lapasset, E. 1995, A\&AS, 109, 375

Ann, H. B., Lee, M. G., Chun, M. Y., Kim, S.-L., Jeon, Y.-B., Park, B.-G., Yuk, I.-S., Sung, H, \& Lee, S. H. 1999, Journal of Korean Astronomical Society, 32, 7

Anthony-Twarog, B. J., Twarog, B. A., Kaluzny, J., \& Shara, M. M. 1990, AJ, 99, 1504

Bertelli, G., Bressan, A., Chiosi, C., Fagotto, F., \& Nasi, E. 1994, A\&AS, 106, 275

Bessell, M. S. 2001, private communications

Bonifazi, A., Pecci, A. F., Romeo, G., \& Tosi, M. 1990, MNRAS, 245, 15

Dean, J. F., Warren, P. R., \& Cousins, A. W. J. 1978, MNRAS, 183, 569

Demarque, P., Sarajedini, A., \& Guo, X.-J. 1994, ApJ, 426

Deutschman, W., Davi, R. J., \& R. E. Schild, 1976, ApJS, 30, 97

Janes, K. A., \& Phelps, R. L. 1994, AJ, 108, 1773

Johnson, H. L., \& Morgan, W. W. 1953, ApJ, 117, 313

Kilkenny, D., van Wyk, F., Roberts, G., Marang, F., \& Cooper, D. 1998, MNRAS, 294, 93

Landolt, A. U. 1992, AJ, 104, 340 (COCD)

Lee, S. H., Ann, H. B., \& Kang, Y.-W. 1999, Publication of Korean Astronomical Society, 14,61

Lucke, P. B. 1978, A\&A, 64, 367

Lyngå, G. 1987, Catalog of Open Cluster Data, Starsbourg, Centre de Donnees Stellaires. 5 th ed. 
Maeder, A., \& Meynet, G. 1991, A\&AS, 89, 451

Menzies, J. W., Marang, F., Laing, J. D., Coulson, I. M., \& Caldwell, J. A. R. 1991, MNRAS, 248, 642

Pandey, A. K., Durgapal, A. K., Bhatt, B. C., Mohan, V., \& Mahra, H. S. 1997, A\&AS, 122,111

Phelps, R. L., \& Janes, K. A. 1993, AJ, 106, 1870

Phelps, R. L., \& Janes, K. A. 1994, ApJS, 90, 31

Scalo, J, 1986, Fund. Cos. Phys., 11, 144

Schmidt-Kaler, Th. 1982, in Numerical Data \& Functional Relationships in Science \& Technology, Vol.2, Springer-Verlag, Berlin

Sung, H., \& Bessell, M. S. 2000, PASA, 17, 244 
Fig. 1.- Annfig1.gif

A mosaic map of grayscale $V$-band images of 12 open clusters. The circles represent the angular diameters of the clusters given in the COCD (Lyngå 1987). The size of each box is $11 .^{\prime} 6 \times 11 . ' 6$. North is up and east is to the left.

Fig. 2.- Annfig2.gif

Observational errors in the present photometry. The error bars represent the standard deviations of observational errors for stars in each magnitude bin of 0.5 mag. The large error bars for the brighter stars are caused by the small number of stars in the magnitude bin.

Fig. 3.- Annfig3.gif

Photometric diagrams for the 12 open clusters. The dashed and solid lines in the color-color diagrams represent the theoretical ZAMS of the Padova isochrones(Bertelli et al. 1994). The solid lines in CMDs are the best fit isochrones.

Fig. 4.- Annfig4.gif

The cleaned CMDs of the 6 open clusters that are supposed to have giant members. The best fit isochrones shifted according to the derived reddening and distance moduli are plotted. 
Table 1: Basic parameters of the 12 open clusters.

\begin{tabular}{|c|c|c|c|c|c|c|}
\hline Cluster & R.A.(2000.) & DEC.(2000.) & 1 & $\mathrm{~b}$ & Trumpler class & Angular Diameter \\
\hline Be 104 & $\begin{array}{lll}0 & 328.1\end{array}$ & 633542.4 & 117.63 & 1.22 & II $1 \mathrm{p}$ & $3 .^{\prime} 0$ \\
\hline Be 60 & 01741.4 & 605740.3 & 118.85 & -1.64 & III $1 \mathrm{p}$ & $3 .^{\prime} 0$ \\
\hline King 15 & 03255.8 & 615133.8 & 120.75 & -0.95 & IV $2 \mathrm{p}$ & $3 .{ }^{\prime} 0$ \\
\hline NGC 381 & $\begin{array}{lll}1 & 8 & 19.9\end{array}$ & $6135 \quad 2.1$ & 124.94 & -1.22 & III $1 \mathrm{~m}$ & $6 .^{\prime} 0$ \\
\hline Be 64 & 2212.8 & 655348.3 & 131.92 & 4.60 & II $1 \mathrm{~m}$ & $2 .^{\prime} 0$ \\
\hline King 6 & $328 \quad 3.5$ & 562730.1 & 143.35 & -0.07 & II $2 \mathrm{~m}$ & $10 .^{\prime} 0$ \\
\hline NGC 1348 & 33351.7 & $5126 \quad 9.5$ & 146.94 & -3.71 & III $2 \mathrm{~m}$ & $5 .^{\prime} 0$ \\
\hline Be 12 & 44437.4 & 424038.3 & 161.68 & -1.99 & II $1 \mathrm{~m}$ & $4 .^{\prime} 0$ \\
\hline Be 70 & 52544.6 & 415344.6 & 166.90 & 3.58 & III $1 \mathrm{~m}$ & $6 .^{\prime} 0$ \\
\hline Be 23 & 63328.5 & 203247.0 & 192.61 & 5.44 & III $1 \mathrm{~m}$ & $4 .{ }^{\prime} 0$ \\
\hline NGC 2259 & 63834.4 & 105324.1 & 201.79 & 2.12 & II $1 \mathrm{p}$ & $3 .^{\prime} 0$ \\
\hline NGC 2304 & 6550.9 & $18 \quad 114.1$ & 197.17 & 8.87 & II $1 \mathrm{~m}$ & $3 .^{\prime} 0$ \\
\hline
\end{tabular}


Table 2: Observational $\log$ of the 12 open clusters.

\begin{tabular}{|c|c|c|c|c|c|}
\hline Cluster & Filter & $\mathrm{T}_{\exp }$ & Cluster & Filter & $\mathrm{T}_{\exp }$ \\
\hline \multirow[t]{4}{*}{ Be 104} & $\mathrm{U}$ & $200 s \times 4$ & Be 60 & $\mathrm{U}$ & $200 s \times 3$ \\
\hline & $\mathrm{B}$ & $200 s \times 3,50 \mathrm{~s}$ & & $\mathrm{~B}$ & $200 s \times 4$ \\
\hline & $\mathrm{V}$ & $100 s \times 3,10 \mathrm{~s}$ & & $\mathrm{~V}$ & $100 s \times 3,50 \mathrm{~s}$ \\
\hline & I & $50 s \times 3,5 \mathrm{~s}$ & & I & $50 s \times 3$ \\
\hline \multirow[t]{4}{*}{ King 15} & $\mathrm{U}$ & $200 s \times 3$ & NGC 381 & $\mathrm{U}$ & $900 \mathrm{~s}, 90 \mathrm{~s}$ \\
\hline & $\mathrm{B}$ & $200 s \times 3,20 \mathrm{~s}$ & & B & $400 \mathrm{~s}, 20 \mathrm{~s}$ \\
\hline & $\mathrm{V}$ & $100 s \times 3,10 \mathrm{~s}$ & & $\mathrm{~V}$ & $130 \mathrm{~s}, 10 \mathrm{~s}$ \\
\hline & $\mathrm{I}$ & $50 s \times 3,5 \mathrm{~s}$ & & I & $50 \mathrm{~s}, 5 \mathrm{~s}$ \\
\hline \multirow[t]{4}{*}{ Be 64} & $\mathrm{U}$ & $900 \mathrm{~s}$ & King 6 & $\mathrm{U}$ & $200 s \times 3$ \\
\hline & $\mathrm{B}$ & $900 \mathrm{~s}$ & & $\mathrm{~B}$ & $200 s \times 3,20 \mathrm{~s}$ \\
\hline & $\mathrm{V}$ & $400 \mathrm{~s}$ & & $\mathrm{~V}$ & $100 s \times 3,10 \mathrm{~s}$ \\
\hline & I & $150 \mathrm{~s} 30 \mathrm{~s}$ & & I & $50 s \times 3,5 \mathrm{~s}$ \\
\hline \multirow[t]{4}{*}{ NGC 1348} & $\mathrm{U}$ & $200 s \times 4$ & Be 12 & $\mathrm{U}$ & $900 \mathrm{~s}$ \\
\hline & $\mathrm{B}$ & $200 s \times 3,20 \mathrm{~s}$ & & $\mathrm{~B}$ & $900 \mathrm{~s}$ \\
\hline & $\mathrm{V}$ & $100 s \times 3,10 \mathrm{~s}$ & & $\mathrm{~V}$ & $400 \mathrm{~s}$ \\
\hline & I & $50 s \times 3,5 \mathrm{~s}$ & & $\mathrm{I}$ & $150 \mathrm{~s}, 30 \mathrm{~s}$ \\
\hline \multirow[t]{4}{*}{ Be 70} & $\mathrm{U}$ & $900 \mathrm{~s}$ & Be 23 & $\mathrm{U}$ & $200 s \times 3$ \\
\hline & $\mathrm{B}$ & $900 \mathrm{~s}, 90 \mathrm{~s}$ & & $\mathrm{~B}$ & $200 s \times 3,20 \mathrm{~s}$ \\
\hline & $\mathrm{V}$ & $400 \mathrm{~s}, 40 \mathrm{~s}$ & & $\mathrm{~V}$ & $100 s \times 3,10 \mathrm{~s}$ \\
\hline & I & $150 \mathrm{~s}, 12 \mathrm{~s}$ & & I & $50 s \times 3,5 \mathrm{~s}$ \\
\hline \multirow[t]{4}{*}{ NGC 2259} & $\mathrm{U}$ & $200 s \times 3$ & NGC 2304 & $\mathrm{U}$ & $900 \mathrm{~s}$ \\
\hline & $\mathrm{B}$ & $200 s \times 3,20 \mathrm{~s}$ & & $\mathrm{~B}$ & $900 \mathrm{~s}, 90 \mathrm{~s}$ \\
\hline & $\mathrm{V}$ & $100 s \times 3,10 \mathrm{~s}$ & & $\mathrm{~V}$ & $300 \mathrm{~s}, 30 \mathrm{~s}$ \\
\hline & $\mathrm{I}$ & $50 s \times 3,5 \mathrm{~s}$ & & $\mathrm{I}$ & $80 \mathrm{~s}, 7 \mathrm{~s}$ \\
\hline
\end{tabular}


Table 3: Physical parameters of the 12 open clusters.

\begin{tabular}{ccccc}
\hline \hline Cluster & $E(B-V)$ & $(m-M)_{0}$ & {$[\mathrm{Fe} / \mathrm{H}]$} & Age $(\mathrm{Myr})$ \\
\hline Be 104 & $0.45 \pm 0.05$ & $13.2 \pm 0.2$ & $0.07 \mathrm{dex}$ & $790 \pm 160$ \\
Be 60 & $0.86 \pm 0.05$ & $13.2 \pm 0.2$ & $0.07 \mathrm{dex}$ & $160 \pm 60$ \\
King 15 & $0.70 \pm 0.12$ & $12.5 \pm 0.4$ & $0.07 \mathrm{dex}$ & $250 \pm 50$ \\
NGC 381 & $0.40 \pm 0.10$ & $10.3 \pm 0.3$ & $0.07 \mathrm{dex}$ & $320 \pm 70$ \\
Be 64 & $1.05 \pm 0.15$ & $13.0 \pm 0.5$ & $-0.61 \mathrm{dex}$ & $1000 \pm 210$ \\
King 6 & $0.50 \pm 0.10$ & $9.7 \pm 0.4$ & $0.46 \mathrm{dex}$ & $250 \pm 50$ \\
NGC 1348 & $1.02 \pm 0.05$ & $11.3 \pm 0.3$ & $0.07 \mathrm{dex}$ & $130 \pm 50$ \\
Be 12 & $0.70 \pm 0.15$ & $12.5 \pm 0.5$ & $0.07 \mathrm{dex}$ & $4000 \pm 820$ \\
Be 70 & $0.48 \pm 0.10$ & $13.1 \pm 0.3$ & $-0.32 \mathrm{dex}$ & $4000 \pm 820$ \\
Be 23 & $0.40 \pm 0.05$ & $14.2 \pm 0.3$ & $0.07 \mathrm{dex}$ & $790 \pm 160$ \\
NGC 2259 & $0.59 \pm 0.05$ & $12.6 \pm 0.3$ & 0.07 dex & $320 \pm 70$ \\
NGC 2304 & $0.10 \pm 0.02$ & $13.0 \pm 0.1$ & $-0.32 \mathrm{dex}$ & $790 \pm 160$ \\
\hline
\end{tabular}


This figure "Annfig1.gif" is available in "gif" format from: http://arxiv.org/ps/astro-ph/0203028v1 
This figure "Annfig2.gif" is available in "gif" format from: http://arxiv.org/ps/astro-ph/0203028v1 
This figure "Annfig3a.gif" is available in "gif" format from: http://arxiv.org/ps/astro-ph/0203028v1 
This figure "Annfig3b.gif" is available in "gif" format from: http://arxiv.org/ps/astro-ph/0203028v1 
This figure "Annfig3c.gif" is available in "gif" format from: http://arxiv.org/ps/astro-ph/0203028v1 
This figure "Annfig4.gif" is available in "gif" format from: http://arxiv.org/ps/astro-ph/0203028v1 